Numer. Math. 19, 400-406 (1972)

(C) by Springer-Verlag 1972

\title{
Correction d'une somme en arithmetique a virgule flottante
}

\author{
M. Pichat
}

Reçu le 8 Juin 1971

Summary. Let $S$ be the sum of given numbers, calculated by normalized floatingpoint arithmetic, the rounding-off law being a truncature with a guard digit. We give an algorithm to obtain all the digits of $S$ as significant digits; very often, one step is enough to provide correction of $S$.

\section{Introduction}

\section{I.1. Notations}

Nous désignerons par:

$b$ la base de la représentation arithmétique considérée

$s$ le nombre des chiffres de mantisse

$\oplus$ et $\ominus$ les opérations d'addition et de soustraction, en arithmétique flottante normalisée. Ces opérations ne sont pas associatives et l'ordre des opérations sera défini de la gauche vers la droite.

Nous considérerons ici pour règle d'arrondi, une règle de troncature avec chiffre de garde.

Rappelons que si $X$ est un nombre à virgule flottante, normalisé, non nul, il est caractérisé par les entiers algébriques $l$ et $p$ tels que:

$$
X=l b^{p-s} \quad \text { avec } \quad b^{s-1} \leqq|l|<b^{s}
$$

en effet, soient $\varepsilon$ le signe, $p$ l'exposant et $a_{i}, 1 \leqq i \leqq s$, les chiffres de la mantisse, de $X$ (le chiffre de tête $a_{1}$ est non nul pour tout nombre à virgule flottante, normalisé, différent de 0 ).

$$
X=\varepsilon\left(\sum_{k=1}^{s} a_{k} b^{-k}\right) b^{p} \quad \text { donc } \quad l=\varepsilon \sum_{k=1}^{s} a_{k} b^{s-k} .
$$

Nous noterons: $\mathscr{E}(X)=p$ l'exposant de $X$

$[x]$ la partie entière de $x$, si $x \in \mathbb{R}^{+}$.

\section{I.2. Rappels}

Soient $X_{1}=l_{1} b^{p_{1}-s}$ et $X_{2}=l_{2} b^{p_{1}-s}, X_{1} \geqq X_{2}$, deux nombres à virgule flottante, positifs. 
Posons :

$$
\begin{aligned}
& S F=X_{1} \oplus X_{\mathbf{2}} \\
& D F=X_{1} \oplus X_{\mathbf{2}}
\end{aligned}
$$

Ces opérations d'addition et de soustraction, en arithmétique à virgule flottante, normalisée, s'effectuent, pour la règle de troncature indiquée comme suit $[1]$ :

1) $X_{1}$ est exprimé sous la forme $\left(b l_{1}\right) b^{p_{1}-s-1}$, c'est à dire, est écrit comme un nombre normalisé de $(s+1)$ chiffres de mantisse. $X_{2}$ est "approché " sous la forme $\left[l_{2} b^{p_{2}-p_{2}+1}\right] b^{p_{1}-s-1}$ (cette valeur étant toujours exacte si $p_{2}-p_{1}+1 \geqq 0$ ). Cette écriture est, en général, non normalisée.

2) les mantisses qui sont à présent alignées et comportent $s+1$ chiffres, sont ajoutées ou soustraites, puis le résultat obtenu est normalisé et tronqué lorsque nécessaire.

On a donc:

$$
\begin{aligned}
& S F=\left(l_{1}+\left[l_{2} b^{p_{1}-p_{1}}\right]\right) b^{p_{1}-s} \quad \text { si } \mathscr{E}(S F)=\mathscr{E}\left(X_{1}\right), \\
& S F=\left[\frac{l_{1}+\left[l_{2} b^{p_{2}-p_{1}}\right]}{b}\right] b^{p_{1}+1-s} \text { si } \mathscr{E}(S F)=\mathscr{E}\left(X_{1}\right)+1
\end{aligned}
$$

De même:

$$
\begin{aligned}
& D F=\left[\frac{b l_{1}-\left[l_{2} b p_{2}-p_{1}+1\right.}{b}\right] b^{p_{1}-s} \quad \text { si } \quad \mathscr{E}(D F)=\mathscr{E}\left(X_{1}\right) \text {, } \\
& D F=\left(b l_{1}-\left[l_{2} b^{p_{1}-p_{1}+1}\right]\right) b^{p_{1}-s-1} \quad \text { si } \quad \mathscr{E}(D F)<\mathscr{E}\left(X_{1}\right)
\end{aligned}
$$

(on a exprimé dans ce dernier cas, la valeur de $D F$ et non l'écriture après normalisation qui fait intervenir un cadrage).

\section{I.3. Remarques}

On remarque que l'on ne fait aucune erreur d'opération, en particulier:

$A$ - en soustrayant deux nombres de même exposant

$B$ - en soustrayant deux nombres dont les exposants différent de une unité, à la condition que de plus, $\mathscr{E}(D F)<\mathscr{E}\left(X_{1}\right)$ strictement.

Ce sont ces opérations "sans erreur" qui permettront de définir un algorithme pour l'obtention de sommes algébriques dont tous les chiffres soient significatifs.

\section{Etude et representation des erreurs d'addition en arithmetique a virgule flottante}

\section{II.1. Etude de la somme et de la différence}

$X_{1}$ et $X_{2}$ étant définis comme au $\S \mathrm{I}$, posons:

$$
\begin{aligned}
& S=X_{1}+X_{2}=S F+E, \\
& D=X_{1}-X_{2}=D F+E^{\prime} .
\end{aligned}
$$


Les remarques $A$ et $B$ précédentes permettent de représenter, dans tous les cas, sous réserve de la capacité de la machine, $E$ et $E^{\prime}$ par les nombres à virgule flottante:

$$
\begin{aligned}
E & =-\left(S F \ominus X_{1} \ominus X_{2}\right) \\
E^{\prime} & =-\left(D F \ominus X_{1} \oplus X_{2}\right)
\end{aligned}
$$

On peut noter qu'une expression théorique de ces erreurs serait obtenue en écrivant les divisions:

et $\operatorname{si} p_{2} \neq p_{1}$

$$
\begin{array}{ll}
l_{2}=b^{p_{1}-p_{2}} q_{1}+r_{1} & r_{1} \leqq b^{p_{2}-p_{2}}-1 \\
l_{1}+q_{1}=b q_{2}+r_{2} & r_{2} \leqq b-1
\end{array}
$$

en effet, on a:

$$
\begin{array}{ll}
l_{2}=b^{p_{1}-p_{2}-1} q_{3}+r_{3} & r_{3} \leqq b^{p_{1}-p_{4}-1}-1 \\
b l_{1}-q_{3}=b q_{4}+r_{4} & r_{4} \leqq b-1
\end{array}
$$

$$
\begin{aligned}
& \text { si } \mathscr{E}(S F)=\mathscr{E}\left(X_{1}\right) \quad E=r_{1} b^{p_{2}-s} \quad \text { et } 0 \leqq E<b^{p_{1}-s} \\
& \text { si } \mathscr{E}(S F)=\mathscr{E}\left(X_{1}\right)+1 \quad E=\left(r_{1}+r_{2} b^{p_{1}-p_{1}}\right) b^{p_{2}-s} \quad \text { et } 0 \leqq E<b^{p_{1}+1-s}
\end{aligned}
$$

et pour la différence, lorsque $p_{2} \neq p_{1}\left(E^{\prime}=0\right.$ pour $\left.p_{2}=p_{1}\right)$

$$
\begin{aligned}
& \text { si } \quad \mathscr{E}(D F)=\mathscr{E}\left(X_{1}\right) \quad E^{\prime}=\left(r_{4} b^{p_{1}-p_{1}-1}-r_{3}\right) b^{p_{2}-s} \quad \text { et } \quad-b^{p_{1}-1-s}<E^{\prime}<b^{p_{1}-s} \\
& \text { si } \mathscr{E}(D F)<\mathscr{E}\left(X_{1}\right) \quad E^{\prime}=-r_{3} b^{p_{2}-s} \quad \text { et } \quad-b^{p_{1}-1-s}<E^{\prime} \leqq 0 \text {. }
\end{aligned}
$$

Dans le cas où $\mathscr{E}(D F)<\mathscr{E}\left(X_{1}\right)$, il est important de remarquer que:

$$
p_{2}=p_{1} \quad \text { ou } \quad p_{2}=p_{1}-1 \Rightarrow E^{\prime}=0 .
$$

$p_{2} \leqq p_{1}-1 \Rightarrow \mathscr{E}(D F)=p_{1}-1$ ce qui permettra d'établir la formule (8) ci-dessous.

\section{II.2. Cas d'une somme algébrique de 2 termes}

Soient à présent, $X_{1}$ et $X_{2}$ deux nombres à virgule flottante, de signes quelconques.

On peut supposer $\left|X_{1}\right| \geqq\left|X_{2}\right|$ et les 4 possibilités:

$$
0<X_{2}<X_{1}, \quad X_{1}<X_{2}<0, \quad X_{2}<0<X_{1}, \quad X_{1}<0<X_{2}
$$

se ramènent par changements de variable aux cas précédents.

Désignons par $\xi_{1}$ et $\xi_{2}$ les 2 nombres à virgule flottante, non nuls, de plus petit module, respectivement positif et négatif.

A la condition que si $E \neq 0$, on ait:

$$
0<\xi_{1} \leqq E \text { ou } E \leqq \xi_{2}<0 .
$$

$E$ est représentable par un nombre en virgule flottante. Il vient:

Proposition II.1. Etant donnés 2 nombres $X_{1}$ et $X_{2}$ à virgule flottante tels que $\left|X_{1}\right| \geqq\left|X_{2}\right|$, si $S=X_{1}+X_{2}$ et $S F=X_{1} \oplus X_{2}$, alors l'erreur $E=S-S F$ est 
représentée (sous réserve que $E$ vérifie (7)) par le nombre à virgule flottante:

$$
E=-\left(S F \ominus X_{1} \ominus X_{2}\right) .
$$

Les expressions théoriques écrites permettent de majorer $E$ :

$$
\text { Si } \mathscr{E}(S F)=P \text {, on a: }|E|<b^{P-s} .
$$

\section{Algorithme correcteur d'une somme de $\boldsymbol{N}$ termes}

Soient $N$ nombres à virgule flottante, $X_{i}, 1 \leqq i \leqq N$.

Nous nous proposons d'obtenir, par des calculs effectués en machine, le nombre en virgule flottante le plus près possible de la somme algébrique $S=\sum_{i=1}^{N} X_{i}$.

Posons: $S F=X_{1} \oplus X_{2} \oplus \cdots \oplus X_{N}$

$$
S=S F+E \text {. }
$$

Si $e_{i}$ désigne l'erreur commise lors de la $i^{\text {ème }}$ addition dans le calcul de $S F$, on a immédiatement:

$$
S=S F+\sum_{i=1}^{N-1} e_{i}
$$

Pour corriger $S F$, on ajoutera à la valeur trouvée, la somme des $e_{i}$, calculée en virgule flottante; cette dernière somme étant entachée d'erreur, on pourra effectuer une deuxième correction, et ainsi de suite.

\section{III.1. Définitions}

Changeant légèrement les notations, on définit les suites $\left\{S F^{n}\right\},\left\{e_{i}^{n}\right\}, i=1$, $\ldots, N-1,\left\{F_{i}^{n}\right\}, i=2, \ldots, N-1$ de la façon suivante:

Les premiers termes de chaque suite sont définis par:

$$
\begin{aligned}
F_{2}^{1} & =X_{1} \oplus X_{2} & e_{1}^{1} & =X_{1}+X_{2}-F_{2}^{1} \\
F_{i+1}^{1} & =F_{i}^{1} \oplus X_{i+1} & e_{i}^{1} & =F_{i}^{1}+X_{i+1}-F_{i+1}^{1} \quad \text { pour } i=2, \ldots, N-2 \\
S F^{1} & =F_{N-1}^{1} \oplus X_{N} & e_{N-1}^{1} & =F_{N-1}^{1}+X_{N}-S F^{1} .
\end{aligned}
$$

(Rappelons que, pour calculer $-e_{i}^{1}$ par exemple, on formera: $F_{i+1}^{1} \ominus F_{i}^{1} \Theta X_{i+1}$ ou $F_{i+1}^{\mathbf{1}} \ominus X_{i+1} \ominus F_{i}^{1}$, selon les grandeurs relatives de $\left|F_{i}^{1}\right|$ et $\left|X_{i+1}\right|$.

Puis, on pose:

$$
\begin{aligned}
F_{2}^{n} & =e_{1}^{n-1} \oplus e_{2}^{n-1} & e_{1}^{n} & =e_{1}^{n-1}+e_{2}^{n-1}-F_{2}^{n} \\
F_{i+1}^{n} & =F_{i}^{n} \oplus e_{i+1}^{n-1} & e_{i}^{n} & =F_{i}^{n}+e_{i+1}^{n-1}-F_{i+1}^{n} \quad \text { pour } \quad i=2, \ldots, N-2 \\
S F^{n} & =S F^{n-1} \oplus F_{N-1}^{n} & e_{N-1}^{n} & =S F^{n-1}+F_{N-1}^{n}-S F^{n} .
\end{aligned}
$$

Nous noterons:

$$
P^{n}=\mathscr{E}\left(S F^{n}\right)
$$


Des définitions, il vient:

$$
S=S F^{n}+\sum_{i=1}^{N-1} e_{i}^{n}
$$

\section{III.2. Convergence de l'algorithme}

Examinons le passage du pas $n-1$ au pas $n$.

Soit $Q^{n}$ le plus petit entier tel que:

$$
\sum_{i=1}^{N-1}\left|e_{i}^{n-1}\right|<b^{Q^{n}}
$$

et montrons que nous avons 2 possibilités:

* soit $Q^{n+1}<Q^{n}$

* soit l'algorithme est terminé.

Si $Z_{1}, Z_{2}, \ldots, Z_{P}$ sont des nombres à virgule flottante, remarquons que:

$$
\left|Z_{1} \oplus Z_{2} \oplus \cdots \oplus Z_{k}\right| \leqq \sum_{i=1}^{P}\left|Z_{i}\right| \text { pour } \forall k \leqq P
$$

en raison de l'hypothèse de troncature (et non d'arrondi).

D'après cette remarque et (8)

$$
\left|e_{i}^{n}\right|<\max \left(b^{Q^{n}-s}, \xi\right) \quad(1 \leqq i \leqq N-2) \quad \text { où } \quad \xi=\max \left(\xi_{1},\left|\xi_{2}\right|\right) .
$$

Limitons nous au cas où $2 N \leqq b^{s-1}$, alors:

Si $b^{Q^{n}-s} \geqq \xi$

$$
\sum_{i=1}^{N-1}\left|e_{i}^{n}\right|<(N-2) b^{Q^{n-s}}+b^{P_{n}-s}<\frac{1}{2} b^{Q^{n-1}}+b^{P n-s}
$$

si $b^{P_{n-s}}<\frac{1}{2} b^{Q^{n-1}}$, il vient $Q^{n+1}<Q^{n} ;$ sinon on a $\sum_{i=1}^{N-1}\left|e_{i}^{n}\right|<2 b^{P_{n-s}}$ et seul le dernier bit de la mantisse de $S F^{n}$ peut être entaché d'erreur.

Si l'on avait eu $b^{Q^{n}-s}<\xi$, la majoration serait: $\left|S-S F^{n}\right| \leqq(N-2) \xi+b^{P_{n}-s}$; la précision est donc limitée dans le cas où $S$ est proche de $\xi$ en valeur absolue, et en particulier si $S F^{n}=0$ on fera $P^{n}=-\infty$ dans la majoration écrite.

donc:

Proposition III.2. Si $2 N \leqq b^{s-1}$, il existe un entier $k>0$ tel que:

$$
\left|S-S F^{k}\right|<\operatorname{Max}\left(2 b^{P^{k}-s},(N-2) \xi+b^{P^{k}-s}\right) \quad \text { où } \quad P^{k}=e\left(S F^{k}\right) .
$$

Remarque: portée de la premiere correction:

On a :

$$
\text { si } p=\max \left(\mathscr{E}\left(F_{i}^{\mathbf{1}}\right), P^{1}\right) \quad i=2, \ldots, N-1 .
$$

$$
\left|S-S F^{2}\right|<b^{p+2 y-2 s}+b^{P^{\prime}-s}
$$

où $\gamma$ est le plus petit entier tel que $N \leqq b^{\gamma}$. 


\section{Exemples numeriques}

Nous avons considéré 2 sommes arithmetiques:

$$
\sigma_{1}=\sum_{I=1}^{N} \frac{1}{I} \quad \text { et } \quad \sigma_{2}=\sum_{I=1}^{N} \frac{1}{I^{2}}
$$

et 2 sommes algébriques:

$$
\sigma_{3}=\sum_{I=1}^{N} \frac{(-1)^{I-1}}{I} \text { et } \sigma_{4}=\sum_{I=1}^{N} \frac{(-1)^{I-1}}{I^{2}} .
$$

Dans chaque cas, soient:

$S$ la valeur de la somme effectuée par une IBM 360-30

$S C$ la valeur de cette somme corrigée par le procédé indiqué (une seule correction)

$S D$ la valeur de la somme effectuée en double précision.

Les calculs ont été faits pour $N$ variant de 100 à 500, par pas de 100 .

\begin{tabular}{lllllll}
\hline & $N$ & 100 & 200 & 300 & 400 & 500 \\
\hline$\sigma_{1}$ & $S$ & 5,187340 & 5,877946 & 6,282538 & 6,569756 & 6,792601 \\
& $S C$ & 5,187377 & 5,878030 & 6,282662 & 6,569928 & 6,729822 \\
& $S D$ & 5,187377 & 5,878030 & 6,282663 & 6,569929 & 6,792822 \\
$\sigma_{2}$ & $S$ & 1,634939 & 1,639858 & 1,641470 & 1,642253 & 1,642706 \\
& $S C$ & 1,634983 & 1,639946 & 1,641605 & 1,642437 & 1,642936 \\
& $S D$ & 1,634983 & 1,639946 & 1,641606 & 1,642437 & 1,642936 \\
$\sigma_{3}$ & $S$ & 0,6881702 & 0,6906486 & 0,6914758 & 0,6918888 & 0,6921354 \\
& $S C$ & 0,6881722 & 0,6906534 & 0,6914833 & 0,6918988 & 0,6921481 \\
& $S D$ & 0,6881722 & 0,6906534 & 0,6914833 & 0,6918987 & 0,6921482 \\
$\sigma_{4}$ & $S$ & 0,8224151 & 0,8224491 & 0,8224530 & 0,8224527 & 0,8224512 \\
& $S C$ & 0,8224175 & 0,8224546 & 0,8224615 & 0,8224639 & 0,8224650 \\
& $S D$ & 0,8224175 & 0,8224545 & 0,8224614 & 0,8224639 & 0,8224650 \\
\hline
\end{tabular}

La lecture des résultats montre que tous les chiffres de la somme corrigée $S C$ sont significatifs, une éventuelle différence entre le derniers chiffres de $S C$ et $S D$ provenant de la conversion des résultats en décimal, comme nous l'avons vérifié en effectuant la sortie de $S, S C, S D$ en hexadécimal.

\section{Conclusion et generalisation}

Dans les cas où il est nécessaire d'obtenir des résultats extrêmement précis, la méthode exposée ci-dessus peut être employée, soit seule avec un nombre suffisant d'itérations, soit combinée à la double précision pour rendre plus rapidement significatifs tous les chiffres du résultat.

Nous avons effectué l'étude précédente avec l'hypothèse que la règle d'arrondi définissant l'arithmétique à virgule flottante, était une règle de troncature avec chiffre de garde. Les résultats obtenus peuvent se formaliser et se généraliser [2], avec quelques modifications à de nombreuses arithmétiques à virgule flottante. 
Ainsi:

1) Si l'addition en virgule flottante est définie de même qu'en I, mais si, lors du cadrage final, on utilise, au lieu de la troncature, les possibilités d'arrondi (un nombre étant arrondi au nombre flottant de valeur absolve supérieure si le $(s+1)^{\text {ième }}$ chiffre de sa mantisse est supérieur ou égal à $\left.b / 2\right)$, on a les résultats suivants:

Dès que $E$ vérifie (7), $E$ est représentable par un nombre à virgule flottante et si $b=2$ ou $b=3, E=-\left(S F \ominus X_{1} \ominus X_{2}\right)$; cette expression n'est plus valable pour une base quelconque, et doit être remplacée par une expression plus compliquée, mais une condition suffisante de convergence de l'algorithme défini en III est encore établie de la même façon.

2) Si la règle d'arrondi définissant l'addition, est une règle de troncature sans chiffre de garde:

L'erreur $E$ n'est plus donnée par l'expression - $\left(S F \ominus X_{1} \ominus X_{2}\right)$; cependant, dès que $E$ vérifie (7), $E$ est représentable à l'aide d'opérations en virgule flottante, et (8) devient:

$$
|E|<b^{P-s} \quad \text { où } \quad P=\max \left\{\mathscr{E}(S F), \mathscr{E}\left(X_{1}\right), \mathscr{E}\left(X_{2}\right)\right\} \text {. }
$$

La convergence de l'algorithme de correction d'une somme est dans ce cas, assurée des que $N \leqq b^{s-2}$.

\section{Références}

1. I.B.M.: Systems réference library. I.B.M. System/360. Principles of operations, 1968, pp. 41-50.

2. Pichat, M.: Séminaire d'analyse numérique Mai, 1972. Université scientifique et médicale de Grenoble I.

\section{Pichat}

Conservatoire National des Arts et Métiers 292, rue Saint-Martin - Paris $3 \mathrm{e}$

Service de Mathématiques appliquées

Cedex 53, 38 - Grenoble - Gare

France 